\title{
Rechtsprechung
}

\section{Kunstfotografien und schweizerische Einfuhrsteuer}

Tribunale federale, Urteil vom 21. Januar 2008 - [T 0/2] 2A.372/2006

\section{Art. 74 Abs. 1 Ziff. 3 MWStG wird auf Kunstfotografien nicht entsprechend angewendet. Kunstfotografien sind} nicht von der Einfuhrsteuer befreit. (Leitsätze der Redaktion)

\section{$[\ldots]$}

\section{Sachverhalt:}

- A. Die X. AG ist u.a. auf den Import und Export von Kunstwerken spezialisiert. Sie meldete am 7. März 2002 beim Zollamt Zürich vier Sendungen mit Kunstfotografien zur abgabefreien Einfuhr an. Das Zollamt lehnte dies ab und erhob mit Einfuhrzoll- und Mehrwertsteuerausweisen vom 18. März 2002 die Einfuhrsteuer zum Normalsatz von insgesamt Fr. 10'570.05 und den Zollbetrag von Fr. 158.65. Am 8. Juli 2002 bestätigte die Zollkreisdirektion Schaffhausen die Zoll- und Einfuhrabgabe. Eine Beschwerde der X. AG wies die Oberzolldirektion mit Entscheid vom 5. Mai 2003 ab.

B. Die X. AG beschwerte sich bei der Eidgenössischen Zollrekurskommission. Diese wies mit Entscheid vom 16. Mai 2006 die Beschwerde ab. Zur Begründung führte sie im Wesentlichen aus, die zollfreie Einfuhr sei im Zollgesetz abschließend geregelt. Art. 74 des Mehrwertsteuergesetzes beschränke die steuerbefreite Einfuhr auf Werke von Kunstmalern und Bildhauern, die durch diese eingeführt würden. Für Werke der Fotokunst sei weder eine Zollbefreiung noch die Befreiung von der Einfuhrsteuer vorgesehen. Die Zollrekurskommission nahm ferner zu den internationalen Verpflichtungen der Schweiz Stellung und legte dar, dass das von der Beschwerdeführerin angeführte „UnescoÜbereinkommen" gemäß seinem Anhang auf Kunstfotografien keine Anwendung finde.

C. Mit Verwaltungsgerichtsbeschwerde vom 15. Juni 2006 beantragt die X. AG dem Bundesgericht, es seien der Entscheid der Eidgenössischen Zollrekurskommission vom 16. Mai 2006, der Entscheid der Oberzolldirektion vom 5. Mai 2003 sowie die Zollausweise aufzuheben; die Einfuhren seien als nicht zoll- und mehrwertsteuerpflichtig zu erklären. Der Betrag von insgesamt Fr. 10`728.70 sei der Beschwerdeführerin zurückzuerstatten.

D. Die Oberzolldirektion beantragt, die Beschwerde kostenfällig abzuweisen. Die Eidgenössische Zollrekurskommission verzichtete auf eine Vernehmlassung.

\section{Erwägungen:}

1.1. Am 1. Januar 2007 trat das Bundesgesetz über das Bundesgericht vom 17. Juni 2005 (BGG; SR 173.110) in Kraft. Da der angefochtene Entscheid der Eidgenössischen Zollrekurskommission vor dem 1. Januar 2007 erging, richtet sich das vorliegende Verfahren noch nach den Bestimmungen des Bundesgesetzes über die Organisation der Bundesrechtspflege (OG) vom 16. Dezember 1946 samt den diesbezüglichen Ausführungsbestimmungen (vgl. Art. 132 Abs. 1 BGG). Die Verwaltungsgerichtsbeschwerde gegen den Entscheid der Eidgenössischen Zollrekurskommission betreffend Zollabgabe und Einfuhrsteuer ist zulässig (Art. 97 Abs. 1 und 98 lit. e OG). Als unzulässig erweist sich indessen der Antrag, der Entscheid der Oberzolldirektion und die Zollausweise seien aufzuheben; diese Verfügungen sind durch den Entscheid der Eidgenössischen Zollrekurskommission ersetzt worden (sog. Devolutiveffekt). Sie gelten aber als inhaltlich mitangefochten (vgl. BGE 133 || 292 E. 1.5, 129 || 438 E. 1).

1.2. Gemäss Art. 108 Abs. 2 OG hat die Beschwerde unter anderem die Begehren und deren Begründung zu enthalten. An die Begründung werden nicht hohe Anforderungen gestellt, doch hat sie in minimaler Form sachbezogen zu sein (BGE 131 II 449 E. 1.3, 470 E. 1.3). Obschon die Beschwerdeführerin mit der vorliegenden Beschwerde eine Zollbefreiung verlangt, begründet sie das zollrechtliche Begehren nicht. Auf den Antrag ist daher nicht einzutreten.

2. Der Mehrwertsteuer unterliegen grundsätzlich alle durch eine steuerpflichtige Person im Inland getätigten Umsätze, soweit sie nicht ausdrücklich von der Steuer ausgenommen sind. Als steuerpflichtige Umsätze gelten nach Art. 5 lit. a-d des Bundesgesetzes über die Mehrwertsteuer vom 2. September 1999 (MWSTG, SR 641.20) die im Inland gegen Entgelt erbrachten Dienstleistungen und Lieferungen von Gegenständen, der Eigenverbrauch im Inland sowie der Bezug von Dienstleistungen aus dem Ausland.

Die Steuer auf den Umsätzen im Inland wird im Gesamtsystem der Umsatzsteuer ergänzt durch eine Einfuhrsteuer (Art. 72 ff. MWSTG). Ohne die Einfuhrsteuer würden die aus dem Ausland eingeführten Gegenstände gegenüber den inländischen Erzeugnissen bevorteilt, weshalb auch die Einfuhr von Gegenständen ins Inland zu besteuern ist (Dieter Metzger, Kurzkommentar zum Mehrwertsteuergesetz, Muri/Bern 2000, N 1 zu 
Art. 72). Steuerobjekt ist gemäss Art. 73 MWSTG die Einfuhr von Gegenständen. Darunter ist das Verbringen von Gegenständen ins Zollgebiet zu verstehen, wie aus dem Verweis von Art. 72 MWSTG auf die Zollgesetzgebung folgt. Steuerpflichtiger ist der Zollzahlungspflichtige. Steuerobjekt und Steuersubjekt entsprechen sich bei der Steuer auf den Inlandumsätzen und der Einfuhrsteuer somit nicht.

3. Art. 74 Abs. 1 MWSTG enthält die Liste der Gegenstände, die bei der Einfuhr von der Steuer befreit sind. Nach Ziffer 3 sind von der Einfuhrsteuer befreit: „3. Kunstwerke, die von Kunstmalern und Bildhauern persönlich bearbeitet und von ihnen selbst oder in ihrem Auftrag ins Inland verbracht wurden, mit Ausnahme des Entgelts nach Artikel 76 Absatz 1 Buchstabe d“. Diese haben bei der Einfuhr lediglich die Steuer auf allfälligen Arbeiten, die sie an ihrem Kunstwerk im Ausland haben besorgen lassen, zu entrichten (Art. 76 Abs. 1 lit. d MWSTG). Die Einfuhr dieser Kunstwerke durch andere Personen unterliegt der Steuer. Die Liste der steuerbefreiten Einfuhren des Art. 74 MWSTG ist abschließend, weil auch Ausnahmen von der Steuer einer gesetzlichen Grundlage bedürfen (Peter Locher, Legalitätsprinzip im Steuerrecht, ASA 60 S. 13 f.; BGE 103 la 505 E. 3b). Werke der Fotokunst sind in Art. 74 MWSTG nicht erwähnt. Diese unterliegen somit der Einfuhrsteuer. Bei einer anschließenden Lieferung solcher Kunstwerke kann aber die Einfuhrsteuer unter den Voraussetzungen von Art. 38 Abs. 1 lit. c MWSTG als Vorsteuer abgezogen werden.

Die Beschwerdeführerin macht geltend, beim Begriff „Kunstwerk“ in Art. 74 Abs. 1 Ziff. 3 MWSTG handle es sich um einen "offenen Begriff". Dieser erfasse bei richtiger Auslegung (was die Beschwerdeführerin in ihrer Beschwerdeeingabe ausführlich darlegt) auch Werke der Fotokunst. Die Beschwerdeführerin verweist besonders auf Art. 18 Ziff. 16 MWSTG, der bei der Steuer auf Inlandumsätzen „kulturelle Dienstleistungen und Lieferungen von Gegenständen durch deren Urheberinnen und Urheber wie Schriftsteller, Komponisten, Filmschaffende, Kunstmaler, Bildhauer ..." von der Steuer ausnimmt, sowie auf Art. 2 Abs. 2 lit. g des Bundesgesetzes vom 9. Oktober 1992 über das Urheberrecht und verwandte Schutzrechte (URG, SR 231.1), wonach als Werke auch: "g. fotografische, filmische und andere visuelle oder audiovisuelle Werke" gelten. Nach Ansicht der Beschwerdeführerin sind diese Bestimmungen bei der Einfuhrsteuer analog heranzuziehen, so dass Art. 74 Abs. 1 Ziff. 3 MWSTG - obwohl darin nicht ausdrücklich erwähnt - auch die Einfuhr von Kunstfotografien durch den Urheber von der Einfuhrsteuer befreit. Wie es sich damit verhält, ist im Folgenden zu prüfen.

4.1. Ausgangspunkt jeder Gesetzesauslegung bildet der Wortlaut der Bestimmung. Ist der Text nicht ganz klar und sind verschiedene Auslegungen möglich, so muss nach seiner wahren Tragweite gesucht werden unter Berücksichtigung aller Auslegungselemente. Abzustellen ist namentlich auf die Entstehungsgeschichte der Norm und ihren Zweck sowie auf die Bedeutung, die der Norm im Kontext mit den anderen Bestimmungen zukommt. Das Bundesgericht hat sich bei der Auslegung von Erlassen stets von einem Methodenpluralismus leiten lassen und hat nur dann allein auf den Wortlaut abgestellt, wenn sich daraus zweifelsfrei die sachlich richtige Lösung ergibt (BGE 131 II 562 E. 3.5, 697 E. 4.1, 710 E. 4.1; 130 || 65 E. 4.2; 125 II 192 E. 3a S. 196 mit Hinweisen).

Die Auslegung kann ergeben, dass das Gesetz lückenhaft ist. Eine echte Gesetzeslücke liegt vor, wenn der Gesetzgeber etwas zu regeln unterlassen hat, was er hätte regeln sollen, und dem Gesetz auch durch Auslegung keine Vorschrift entnommen werden kann, welche eine Antwort auf die Frage gibt. Von einer unechten oder rechtspolitischen Lücke ist demgegenüber die Rede, wenn dem Gesetz zwar eine Antwort, aber keine befriedigende, zu entnehmen ist, namentlich wenn die vom klaren Wortlaut geforderte Subsumtion eines Sachverhalts in der Rechtsanwendung teleologisch als unhaltbar erscheint. Echte Lücken zu füllen, ist dem Richter aufgegeben, unechte zu korrigieren, ist ihm grundsätzlich verwehrt, es sei denn, die Berufung auf den als maßgeblich erachteten Wortsinn der Norm stelle einen Rechtsmissbrauch dar (BGE 132 III 707 E. 2; 131 II 562 E. 3.5; 128 I 34 E. 3b S. 42; 121 III 219 E. 1d/aa S. 225 f.). Hat der Gesetzgeber eine Rechtsfrage nicht übersehen, sondern stillschweigend (im negativen Sinn) mitentschieden, so handelt es sich um ein qualifiziertes Schweigen und ist kein Platz für eine richterliche Lückenfüllung (BGE 132 III 470 E. 5.1; 129 V 1 E. 4.1.1). Die gleichen Grundsätze gelten für das Steuerrecht (BGE 131 II 562 E. 3.5 S. 568 mit Hinweisen).

4.2. Der Wortlaut von Art. 74 Abs. 1 Ziff. 3 MWSTG ist in allen drei Sprachen klar. Von der Einfuhrsteuer befreit sind Kunstwerke von Kunstmalern und Bildhauern (artistes-peintres ou sculpteurs, pittori e scultori), die durch diese persönlich bearbeitet worden sind und von diesen selbst oder in deren Auftrag eingeführt werden. Die Werke von Fotografen werden in Art. 74 MWSTG nicht erwähnt.

Bereits unter der Warenumsatzsteuer waren „Kunstmaler und Bildhauer für die von ihnen persönlich hergestellten Kunstwerke" nicht als Grossisten steuerpflichtig (Art. 11 Abs. 1 lit. d WUStB). Diese Steuerbefreiung galt nach der Rechtsprechung für die Steuer auf dem Warenumsatz im Inland wie auch für die Einfuhrsteuer (BGE $118 \mathrm{lb} 187 \mathrm{ff}$.). Kunstmaler und Bildhauer hatten somit ihre Werke, die sie in die Schweiz einführten, nicht zu versteuern (Dieter Metzger, Handbuch der Warenumsatzsteuer, Muri/Bern 1992, S. 105 Rz. 205). Das spricht dafür, den Begriff "Kunstwerke, die von Kunstmalern und Bildhauern persönlich bearbeitet ... wurden" in Art. 74 Abs. 1 Ziff. 3 MWSTG gleich auszulegen wie bereits unter der Warenumsatzsteuer. Es gibt keinerlei Hinweise, dass für die Mehrwertsteuer der Gesetzgeber dem Begriff eine andere, neue Bedeutung beilegen wollte.

Auch wenn die klassische Einteilung der bildenden Kunst in Malerei, Bildhauerei, Grafik und Architektur durch neue Ausdrucksformen und Techniken einem Wandel unterliegt und im vergangenen Jahrhundert namentlich auch die Fotografie als Teil der bildenden Kunst anerkannt wurde, lassen sich die einzelnen Kategorien - im Kernbereich - doch auseinanderhalten: 
Darüber, was das Werk eine Kunstmalers, eines Bildhauers, eines Fotografen ist, besteht eine allgemeine Anschauung. Nach dieser Auffassung bildet insbesondere auch die Fotografie eine eigenständige Kunstform. Wohl kann in Grenzbereichen die Unterscheidung bzw. Abgrenzung Schwierigkeiten bereiten. So mag fraglich sein, ob eine kunstmalerisch nachbearbeitete oder verfremdete Fotografie der Malerei oder der Fotokunst zuzuweisen sei. Um solche Grenzfälle geht es vorliegend indessen nicht. Die Beschwerdeführerin behauptet nicht, jedenfalls nicht substantiiert, dass es sich bei den eingeführten Kunstwerken um solche handelt, die richtigerweise oder besser der Malerei zuzuordnen wären.

4.3. Wenn daher die Beschwerdeführerin geltend macht, Werke der Fotografie seien ebenfalls unter die Kunstwerke von Kunstmalern und Bildhauern nach Art. 74 Abs. 1 Ziff. 3 MWSTG zu subsumieren, behauptet sie entweder, es liege eine rechtspolitische Lücke vor, weil dem Gesetz zwar eine Antwort, eine negative, entnommen werden kann, diese aber rechtspolitisch unerwünscht sei. Diese Lücke zu beseitigen vermag indessen wie gesagt nur der Gesetzgeber. Oder dann will die Beschwerdeführerin geltend machen, es handle sich um eine echte Lücke, weil der Gesetzgeber diesen Fall versehentlich zu regeln unterlassen habe bzw. unter teleologischen Gesichtspunkten das Resultat als unvollständig erscheint. Solche Lücken zu füllen, ist dem Richter aufgegeben (vorstehende E. 4.1).

Ob eine echte Lücke vorliegt, ist im Folgenden zu prüfen.

5.1. Die Einfuhrsteuer ergänzt wie ausgeführt die Steuer auf den Umsätzen im Inland. Art. 74 Abs. 1 Ziff. 3 MWSTG kann daher nicht ohne Rücksicht auf die für Inlandumsätze geltenden Vorschriften betrachtet werden. Art. 18 MWSTG enthält die abschließende Liste der von der Steuer ausgenommenen Umsätze im Inland. Gemäß Art. 18 Ziff. 16 MWSTG sind von der Steuer ausgenommen: ,16. kulturelle Dienstleistungen und Lieferungen von Gegenständen durch deren Urheberinnen und Urheber wie Schriftsteller, Komponisten, Filmschaffende, Kunstmaler, Bildhauer sowie von den Verlegern und den Verwertungsgesellschaften zur Verbreitung dieser Werke erbrachten Dienstleistungen". Es handelt sich bei den Steuerausnahmen des Artikels 18 MWSTG um unechte Befreiungen, weil gemäß Art. 17 MWSTG der Vorsteuerabzug nicht geltend gemacht werden kann. Das heißt, die Steuern, die auf den Eingangsmaterialen, Werkzeugen und allfälligen Dienstleistungen lasten, bleiben dem Kunstwerk verhaftet (sog. Taxe occulte). Unter dem Vorbehalt, dass der Künstler nicht für die freiwillige Versteuerung optiert (vgl. Art. 26 Abs. 1 lit. a MWSTG), kann er weder bei der Lieferung im Inland noch bei der Ausfuhr des Kunstwerks die darauf lastenden Vorsteuern zurückverlangen.

Wie eine Durchsicht zeigt, besteuern die umliegenden Länder in der Regel Kunstwerke zu einem reduzierten Satz (Value Added Taxation in Europe, Ernst \& Young Tax Advisers [Herausgeber], IBFD-Publikation Amsterdam, France-106 Ziff. 7.3.1, Germany-151, Italy-161, Netherlands-157). Das bedeutet gleichzeitig, dass beim Export dieser Kunstwerke in einen Nichtmitgliedstaat - wie die Schweiz - der Abzug der ausländischen Vorsteuer geltend gemacht werden kann (Art. 15 Ziff. 2, 17 (3) (b) der Sechsten Richtlinie des Rates vom 17. Mai 1977 zur Harmonisierung der Rechtsvorschriften der Mitgliedstaaten über die Umsatzsteuern, 77/388 EWG; s. auch die analoge schweizerische Regelung, Art. 19 Abs. 2 Ziff. 1 in Verb. mit Art. 38 Abs. 1 lit. c und Abs. 3 MWSTG, und dazu Metzger, Kurzkommentar zum Mehrwertsteuergesetz, a.a.O., N 7 zu Art. 38). Es ist daher folgerichtig, wenn das Mehrwertsteuergesetz die Einfuhr von Kunstwerken grundsätzlich der Einfuhrsteuer unterwirft. Ohne diese Steuer würde auf den eingeführten Kunstwerken keine Steuer (auch keine Taxe occulte) lasten. Der Zweck der Einfuhrsteuer, die Herstellung gleichwertiger Verhältnisse, würde offensichtlich nicht erreicht.

5.2. Bemessungsgrundlage der Einfuhrsteuer bildet das Entgelt oder der Marktwert des Gegenstandes (Art. 76 Abs. 1 lit. $a$ und b MWSTG). Die Erhebung der Einfuhrsteuer auf dem deklarierten Warenwert des Kunstwerks führt zwar zu einer Belastung, die in der Regel höher ist als die Vorsteuer, die auf dem Kunstwerk lasten würde, wenn es im Inland hergestellt worden wäre. Das ist jedoch systembedingt und ergibt sich aus dem Umstand, dass die Steuerobjekte bei der Einfuhrsteuer und der Inlandumsatzsteuer nicht die gleichen sind. Schließt an die Einfuhr eine Lieferung im Inland an oder wird das Kunstwerk wieder ausgeführt, so kann für die Versteuerung optiert werden (Art. 26 Abs. 1 lit. a MWSTG). Damit kann gemäß Art. 38 Abs. 1 lit. c MWSTG die für die Einfuhr deklarierte oder entrichtete Steuer abgezogen werden und werden gleichwertige Verhältnisse hergestellt. Wenn daher der Gesetzgeber die Einfuhr von Kunstwerken durch den Künstler von der Steuer nicht generell (sondern nur punktuell für Kunstmaler und Bildhauer) befreit hat, kann darin keine (echte) Lücke erblickt werden. Dass eine Ordnung unter Umständen nicht immer zweckmäßig sein mag, lässt das Gesetz nicht lückenhaft scheinen. Der Richter darf nur dort eine Lücke bejahen und neue Rechtssätze aufstellen, wo kein Zweifel besteht, dass dem Gesetz eine Norm nicht entnommen werden kann und auch durch analoge Rechtsanwendung sich keine Lösung finden lässt (BGE 94 I 305 E. 2 S. 308 f.).

5.3. Art. 18 Ziff. 16 MWSTG nimmt für die Steuer auf den Umsätzen im Inland die kulturellen Dienstleistungen und Lieferungen von Gegenständen durch Urheber von der Steuer generell aus. Mit dem Begriff des Urhebers knüpft Art. 18 Ziff. 16 MWSTG an das Urheberrechtsgesetz an. Als Werke im Sinne des Urheberrechts gelten auch die von Kunstfotografen geschaffenen fotografischen Werke (Art. 2 Abs. 2 lit. g des Bundesgesetzes über das Urheberrecht und verwandte Schutzrechte, URG, SR 231.1; s. auch Art. 11 Abs. 1 lit. g der Verordnung zum Mehrwertsteuergesetz, MWSTG, SR 641.201). Kunstwerke von Kunstmalern, Bildhauern und Fotografen unterliegen somit hinsichtlich der Inlandumsatzsteuer der exakt gleichen rechtlichen Ordnung. Umsätze solcher Kunstwerke im Inland sind von der Steuer ausgenommen; der Vorsteuerabzug ist ausgeschlossen (Art. 17, 18 Ziff. 16 MWSTG). 
Beim Arbeitsergebnis des Kunstmalers fallen allerdings die Vorsteuern nicht stark ins Gewicht. Die steuerbelasteten Eingangsleistungen beschränken sich im Wesentlichen auf die Arbeitsutensilien und Materialien wie Farben, Kreide, Malgrund usw. Die Miete des Ateliers ist in der Regel von der Mehrwertsteuer ausgenommen (Art. 18 Ziff. 21 und Art. 26 Abs. 1 lit. b MWSTG). Beim Kunstmaler zählt daher die persönliche Leistung weit mehr als das Material (ASA 43 S. 446 E. 2). Höher mag die Vorsteuerbelastung beim Bildhauer sein, weil bei diesem auch die Material- und Werkzeugkosten zu Buch schlagen, doch ist diese Steuerausnahme historisch zu verstehen (vgl. Metzger, Handbuch zur Warenumsatzsteuer, a.a.O., N 201). Würde auf der Einfuhr des Kunstwerks durch den Maler (oder Bildhauer) auf dem Entgelt oder auf dem deklarierten Warenwert die Einfuhrsteuer erhoben, so wäre die eingeführte Ware mit einer Steuer belastet, welche unverhältnismäßig höher ist als die Vorsteuer, die auf dem im Inland hergestellten Kunstwerk lastet. Deshalb befreit Art. 74 Abs. 1 Ziff. 3 MWSTG die Kunstwerke der Maler und Bildhauer von der Einfuhrsteuer (zur ratio dieser Norm, s. auch BGE 118 lb 187 E. 5d S. 194/195).

Kunstfotografen befinden sich demgegenüber nicht in der gleichen Situation. Sie arbeiten mit anderen Arbeitstechniken, für die ihnen andere Berufswerkzeuge zur Verfügung stehen, und stellen auch ein anderes Arbeitsprodukt her. Ihre Arbeitsweise kennzeichnet sich bereits durch einen hohen Technisierungsrad. Es handelt sich um verschiedene Fälle, die auch eine differenzierte Betrachtung rechtfertigen können und welche der Gesetzgeber unterschiedlich behandelt hat. Die Annahme einer Gesetzeslücke, die der Richter zu füllen hätte, verbietet sich unter diesen Umständen.

6. Die Beschwerdeführerin beruft sich auch auf die Vereinbarung über die Einfuhr von Gegenständen erzieherischen, wissenschaftlichen oder kulturellen Charakters, welches für die Schweiz am 7. April 1953 in Kraft getreten ist (SR 0.631.145.141). Gemäß Art. I Ziff. 1 verpflichten sich die Vertragsstaaten, keine Zölle oder andere Abgaben zu erheben für die Einfuhr oder anlässlich der Einfuhr von: a) Büchern, Veröffentlichungen und Dokumenten, die im Anhang A dieser Vereinbarung aufgeführt sind, und b) Gegenständen erzieherischen, wissenschaftlichen oder kulturellen Charakters, die in den Anhängen B, C, D und E dieser Vereinbarung bezeichnet sind. Kunstfotografien werden in den Anhängen A - E weder ausdrücklich erwähnt, noch können sie einem der dort angeführten Erzeugnissen zugeordnet werden, wie die Vorinstanz zutreffend dargelegt hat. Insbesondere lassen sie sich auch nicht unter die in Anhang B genannten Kunstwerke einordnen. Es kann diesbezüglich auf die Ausführungen im angefochtenen Entscheid verwiesen werden. Damit muss nicht entschieden werden, ob diese Vereinbarung unmittelbar anwendbar (self-executing) ist in dem Sinne, dass sich der einzelne Bürger direkt auf die Abkommensbestimmungen berufen und deren Verletzung rügen kann (vgl. BGE 124 IV 23 E. 4a mit Hinweisen).
7. Die Beschwerde ist nach dem Gesagten unbegründet und abzuweisen, soweit darauf einzutreten ist. Dem Verfahrensausgang entsprechend sind die Kosten des bundesgerichtlichen Verfahrens der Beschwerdeführerin aufzuerlegen (Art. 153, 153a, 156 Abs. 1 OG). Ein Anspruch auf Parteientschädigung besteht nicht (Art. 159 Abs. 2 OG).

\section{Demnach erkennt das Bundesgericht:}

1. Die Verwaltungsgerichtsbeschwerde wird abgewiesen, soweit darauf einzutreten ist.

2. Die Gerichtsgebühr von Fr. 2.500.-- wird der Beschwerdeführerin auferlegt.

3. Dieses Urteil wird den Parteien und der Eidgenössischen Zollrekurskommission schriftlich mitgeteilt. 